\title{
Nichtkleinzelliges Lungenkarzinom
}

\section{TITAN-Studie: Erlotinib als gleichwertige Option in der Second-line-Therapie}

In der offen randomisierten Phase-III-Studie TITAN wurde Erlotinib mit einer Chemotherapie in der Second-line-Therapie des fortgeschrittenen nichtkleinzelligen Lungenkarzinoms (NSCLC) verglichen (Ciuleanu T et al., 2010, Chicago Multidisciplinary Symposium in Thoracic Oncology: LBOA5). Prof. Dr. Frank Griesinger, Oldenburg, kommentierte die Ergebnisse der TITAN-Studie in einem Interview.

\begin{abstract}
Herr Professor Griesinger, wie ist die Studie TITAN (Tarceva in Treatment of Advanced NSCLC) in die aktuelle Studienlandschaft beim NSCLC einzuordnen?

Die TITAN-Studie ist die erste prospektiv randomisierte Studie, in der Erlotinib direkt mit den beiden anderen in der Second-line-Therapie zugelassenen Substanzen, Docetaxel und Pemetrexed, verglichen wurde. Diese Studie differenziert sich von anderen Studien wie BR.21 und INTEREST (Iressa Non-small Cell Lung Cancer Trial Evaluating Response and Survival Against Taxotere) dadurch, dass ausschließlich Patienten mit sehr ungünstigen Prognosefaktoren eingeschlossen wurden. Die Patienten hatten eine primäre Progression nach vier Zyklen der Induktionschemotherapie. Die TITAN-Studie gibt uns also Datensicherheit bezüglich second-line Erlotinib, ist aber aufgrund ihres Patientenkollektivs mit anderen Studien nur schwer vergleichbar.
\end{abstract}

\section{Wie bewerten Sie die Ergebnisse in Bezug auf die Wirksamkeit?}

Der primäre Studienendpunkt war das Gesamtüberleben, und das war mit median 5,5 Monaten versus 5,3 Monate vergleichbar zwischen Erlotinib und der Chemotherapie (Hazard-Ratio 0,96). Nach ca. 15 Monaten wurde interessanterweise im Erlotinib-Arm ein et-

\section{Schnellster Post-ASCO 2011}

Unter www.schnellster-post-asco.de gibt es vom 4. bis zum 8. Juni 2011 täglich News vom Annual Meeting der American Society of Clinical Oncology (ASCO) 2011. Renommierte deutsche Onkologen berichten jeden Morgen über die wichtigsten am Vortag präsentierten Studienergebnisse. Die Onko-News sind täglich ab 7 Uhr morgens deutscher Zeit online verfügbar. Die von der HEXAL AG unterstützte Aktion ist ein von der ASCO offiziell lizenzierter Informationsservice. was höheres Plateau erreicht als im Chemotherapie-Arm. Dies bedeutet, dass es eine Patientengruppe gibt, die langfristig stärker von Erlotinib profitiert als von der Chemotherapie. Interessant wäre herauszufinden, welche Patienten das sind. Was die Studie aber sicher zeigt, ist, dass das Gesamtüberleben mit Erlotinib in der Second-line-Therapie bei Patienten mit primär progredienter Erkrankung vergleichbar ist mit dem unter der Chemotherapie. Auch das progressionsfreie Überleben war in beiden Studienarmen nicht signifikant unterschiedlich, wenn auch aufgrund des ungünstigen Risikoprofils der selektionierten Patienten vergleichsweise kurz.

Wichtig bei der Betrachtung des Gesamtüberlebens ist, dass der Anteil der Patienten, die eine Drittlinientherapie erhielten, mit 47\% nach Erlotinib und 43\% nach der Chemotherapie etwa gleich hoch war. Numerisch konnten Patienten aus dem Erlotinib-Arm sogar etwas häufiger eine weitere Therapie erhalten, was für die gute Verträglichkeit spricht.

Wurden bei der Verträglichkeit Unterschiede beobachtet?

Erlotinib war etwas besser verträglich, obwohl Docetaxel und Pemetrexed ebenfalls gut verträgliche Substanzen sind. Unter Erlotinib traten als bekannte Nebenwirkungen der EGFR (Epidermal Growth Factor Receptor)-Inhibitoren Rash und Diarrhö auf, was häufiger als unter den Chemotherapien zu einer Dosismodifizierung führte. Hämatologische Nebenwirkungen traten unter Erlotinib nicht auf. Therapieabbrüche wurden unter der Chemotherapie häufiger beobachtet (4,7\% versus $2,0 \%)$, und es gab mehr Therapie-assoziierte Todesfälle $(5,2 \%$ versus 1,5\%). Der deutlichste Unterschied in Bezug auf die Sicherheit waren weniger schwere Nebenwirkungen im Erlotinib-Arm (10,2\% versus $14,6 \%$ im Chemotherapie-Arm).

Welche Konsequenzen ergeben sich aus diesen Ergebnissen für den Praxisalltag? Wir wissen nun, dass wir einen NSCLC-Patienten mit Progression unter der First-line-Chemotherapie anstatt mit einer Mono-Chemotherapie ebenso wirksam und sicher mit Erlotinib therapieren können: Die Effektivität ist vergleichbar, die Toxizität etwas geringer. Mit Erlotinib ist zudem eine orale Therapie verfügbar, was einige Patienten einer Infusion sicherlich vorziehen. Den Patienten, die mit der primären Chemotherapie nicht gut zurecht gekommen sind, kann mit Erlotinib eine zielgerichtete Therapie angeboten werden, die eben keine Chemotherapie darstellt. Quelle: Information der Roche Pharma AG; Bericht: Dr. Ine Schmale, Limburg.

\section{Trastuzumab plus Vinorelbin} von der AGO mit " $++{ }^{\prime \prime}$ bewertet Die Arbeitsgemeinschaft Gynäkologische Onkologie e.V. (AGO) hat in ihren aktualisierten Leitlinien die Kombination Trastuzumab plus Vinorelbin (Navelbine ${ }^{\circledR}$, Pierre Fabre Pharma $\mathrm{GmbH}$ ) zur First-line-Therapie des HER2positiven, metastasierten Mammakarzinoms mit " ++ " bewertet (Guidelines Breast Version 2011.1). Demnach ist die Kombination für die Patientinnen von großem Vorteil und wird uneingeschränkt von der AGO empfohlen.

\section{Fentanyl-Nasenspray}

PecFent $^{\oplus}$ (Archimedes Pharma Deutschland $\mathrm{GmbH}$ ) kann seit Kurzem in den Dosierungsstärken $100 \mu \mathrm{g}$ bzw. $400 \mu \mathrm{g}$ pro Sprühstoß auch als 12-Flaschen-Packung verordnet werden. Das Fentanyl-Nasenspray ist seit Ende 2010 in Europa zur Behandlung von Tumordurchbruchschmerzen bei Erwachsenen, die eine Basisschmerztherapie mit Opioiden erhalten, verfügbar. Es basiert auf dem patentierten Applikationssystem PecSys ${ }^{\mathrm{TM}}{ }^{\text {, }}$, das die intranasale Fentanyl-Freisetzung optimiert. 